\title{
Transitional Endoplasmic Reticulum ATPase
}

National Cancer Institute

\section{Source}

National Cancer Institute. Transitional Endoplasmic Reticulum ATPase. NCI Thesaurus.

Code C124934.

Transitional endoplasmic reticulum AT Pase (806 aa, $~ 89 \mathrm{kDa}$ ) is encoded by the human VCP gene. This protein is involved in maintaining the Golgi during and after mitosis and the the formation of the transitional endoplasmic reticulum. 